\title{
Rôle du peptide vasoactif intestinal (VIP) dans la stéroïdogenèse et le vieillissement testiculaire
}

\author{
Arnaud LACOMBE ${ }^{1,4}$, Vincent LELIEVRE ${ }^{2,5}$, Charles E. ROSELLI $^{3}$, Jean-Marc MULLER ${ }^{4}$, \\ James A. WASCHEK ${ }^{5}$, Eric VILAIN ${ }^{1}$ \\ 1 Departments of Human Genetics, Pediatrics and Urology, Gonda Center, 695 Charles Young Drive South, Los \\ Angeles, CA 90095-7088 \\ 2 INSERM U676, Hôpital Robert-Debré, 48 bd Sérurier, F-75019 Paris France \\ 3 Department of Physiology and Pharmacology L334, Oregon Health \& Science University, Portland, OR, USA \\ 4 Institut de Physiologie et Biologie Cellulaires, Centre National de la Recherche Scientifique-Unité Mixte de \\ Recherche 6187, Poitiers, France \\ 5 Mental Retardation Research Center, UCLA neurosciences research building, 655 Charles Young Drive South, \\ Los Angeles
}

\section{RESUME}

Des travaux in vitro suggèrent que le VIP pourrait jouer un rôle important au niveau de la biosynthèse de testostérone. Nous avons démontré que des Souris chez lesquelles le gène codant pour le VIP est supprimé montrent des niveaux de testostérone sériques très réduits comparés aux animaux sauvages. Egalement, ces animaux transgéniques présentent une réduction des niveaux de FSH (Follicle-stimulating hormone) à l'âge de quatre mois.

L'étude de la structure testiculaire a révélé une légère augmentation du pourcentage de tubules dégénérés chez les animaux VIP-/- comparés aux animaux sauvages. Les animaux chez lesquels le gène codant pour le VIP a été supprimé, à l'âge de quinze mois, ont des niveaux de stéroïdogenèse bas comparés aux souris sauvages. De manière intéressante, la dégénérescence testiculaire est moins sévère chez les animaux VIP-/- comparée à celles des animaux sauvages.

L'ensemble de ces résultats suggère que : 1) le VIP est un facteur important pour la régulation de la biosynthèse de testostérone et la sécrétion de FSH et 2) VIP régule le vieillissement testiculaire.

Mots clés : neuropeptide, biosynthèse de testostérone, vieillissement testiculaire, Luteinizing Hormone, Follicle Stimulating Hormone

\section{INTRODUCTION}

Le VIP, isolé à partir de duodénum de porc [10], est un peptide composé de 28 acides aminés. Avec son analogue, Ie PACAP (Pituitary adenylate cyclase activating peptide), il appartient à la famille de la sécrétine et du glucagon. L'expression du VIP est ubiquitaire, puisqu'il est produit et sécrété non seulement par l'intestin, mais aussi dans des structures spécifiques du cerveau et bien d'autres tissus périphériques $[4,6,8,10,11]$.

Parmi ses nombreuses activités biologiques, le VIP est décrit comme un peptide aux propriétés neurotrophiques et neuroprotectives bien connues [1, 7]. Des travaux réalisés au cours de la dernière décennie suggèrent qu'il pourrait agir également en tant qu'hormone à action locale [5]. En particulier, le VIP, injecté de façon intracaverneuse, seul ou associé à l'acétylcholine, a été utilisé chez le Rat et l'Humain pour traiter l'impuissance fonctionnelle [13].

Au niveau testiculaire, des études ont démontré qu'in vitro, le traitement de cellules de Leydig par du VIP entraîne une augmentation dose-dépendante de la

Correspondance :

Dr Eric VILAIN - David Geffen School of Medicine at UCLA Gonda Center, Room 6357 - 695 Charles Young Drive South Los Angeles, CA 90095-7088 - Tel (310) 267-2455 - Fax (310) 794-5446 - Email evilain@ucla.edu 
production de testostérone, de progestérone et de prégnénolone [3] suggérant un mode d'action direct du VIP sur les cellules de Leydig. Les effets physiologiques du VIP dans le testicule seraient médiés par son interaction avec le récepteur polyvalent VPAC2 qui a été détecté dès le stade embryonnaire $\mathrm{E} 15.5$, dans les testicules fœtaux.

Basé sur ces résultats, nous avons émis l'hypothèse que ce neuropeptide pouvait être un important régulateur de la biosynthèse de testostérone in vivo. Dans le présent article, nous avons étudié la stéroïdogenèse et le vieillissement testiculaire chez les souris VIP-/- et comparé ces résultats avec ceux précédemment obtenus avec les souris PACAP-I-.

\section{MATÉRIELS ET MÉTHODES}

\section{Caractérisation des niveaux de stéroïdogenèse chez les souris PACAP-I-}

Après avoir anesthésié les animaux, le sang a été collecté et les concentrations sériques en testostérone, $\mathrm{LH}$ (Luteinizing hormone) et FSH (Follicle-stimulating hormone) ont été mesurées par radioimmunoanalyse [9]. Afin de mesurer les niveaux de stéroïdogenèse, les testicules de chaque souris ont été disséqués, pesés, congelés dans de l'azote liquide et homogénéisés dans une solution de TriZol. L'ARN total des tissus a été isolé et des échantillons d'ARN total ont été transcrits de façon inverse en ADN. La quantification de l'expression de StAR (STeroidogenic Acute Regulatory protein) et de 3b-HSD (3?-hydroxysteroid dehydrogenase) a été réalisée par PCR quantitatives en temps réel.

\section{Histopathologie}

Afin de déterminer la structure testiculaire des souris VIP$/-$, des sections de testicules de souris de type sauvage et VIP-/- ont été colorées par la méthode hématoxylineéosine. Les tubules séminifères ont été comptabilisés comme "non-dégénérés" si l'architecture générale montrait une spermatogenèse normale avec toutes les couches de cellules aux différents stades de développement. De manière opposée, ils ont été comptabilisés comme "dégénérés" lorsqu'ils présentaient une déplétion de toutes ou quelques unes des couches de cellules germinales, souvent associée à la présence de vacuolisations au sein des tubules séminifères.

\section{RÉSULTATS}

Lors de cette étude, nous avons démontré que :

\section{Chez les souris VIP-/- de $\mathbf{4}$ mois :}

- Les niveaux sériques de testostérone sont dramatiquement bas comparés aux souris de type sauvage (Figure 1A);
- La stéroïdogenèse, évaluée par les niveaux de testostérone, de StAR, de 3ß-HSD et de P450c17 : 17-hydroxyprogesterone, est diminuée (Figure 1B et $1 \mathrm{C}$ );

- Les niveaux sériques de FSH sont significativement plus bas que chez les souris de type sauvage (Figure $2 \mathrm{~A}$ et $2 \mathrm{~B}$ );

- Il existe une dégénérescence testiculaire modérée comparée aux souris sauvages (Figure $3 \mathrm{~A}, 3 \mathrm{~B}$ et $3 \mathrm{C}$ ).

\section{Chez les animaux âgés de 15 mois :}

- Le testicule de type sauvage présente les signes typiques du vieillissement (dégénérescence des tubules séminifères, épuisement des cellules germinales et présence de vacuolisations au sein des tubules séminifères), tandis que la structure testiculaire est bien mieux conservée chez les animaux VIP -/- (Figure 3A, 3B et 3C);

- Les niveaux sériques de testostérone et la stéroïdogenèse sont bas et constants chez les animaux mutants (Figures 1 et 2 ).

\section{DISCUSSION}

Nous avons tout d'abord observé que les souris VIP-/ont des niveaux sériques de testostérone considérablement diminués par comparaison aux souris de type sauvage. Ce phénomène est associé à une diminution d'expression de StAR et 3ß-HSD. Ce résultat est consistant avec de précédentes études in vitro montrant que le VIP stimule la stéroïdogenèse sur cellules de Leydig de rat en culture [2].

II est intéressant de noter que cette diminution de concentration en testostérone apparaît être plus importante chez les souris VIP-/- que chez les souris PACAP-/- puisque les niveaux enregistrés chez les souris VIP-/- sont encore plus bas que chez les souris PACAP-/-. Puisque des études ont démontré que les souris mutantes VPAC2-/- sont hypofertiles, nous supposons que le récepteur VPAC2 est celui qui est impliqué dans la médiation des effets du VIP dans le testicule.

Le deuxième but de notre étude a été d'analyser les effets à long terme de la réduction de stéroïdogenèse sur la morphologie testiculaire. Nous avons observé une protection contre le vieillissement testiculaire chez les souris mutantes VIP -/- comparées aux souris de type sauvage. Cependant, le degré de protection induit par le manque de VIP n'est pas aussi élevé que celui obtenu avec les souris PACAP KO. A l'âge de 4 mois, les souris VIP-/- ont un niveau sérique de testostérone très bas et un niveau sérique de $\mathrm{FSH}$ significativement réduit, comparés aux souris de type sauvage. Ces deux 


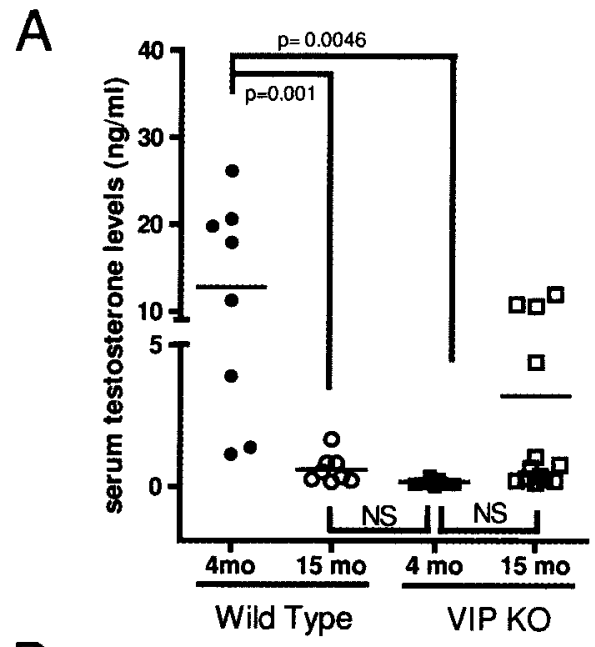

B

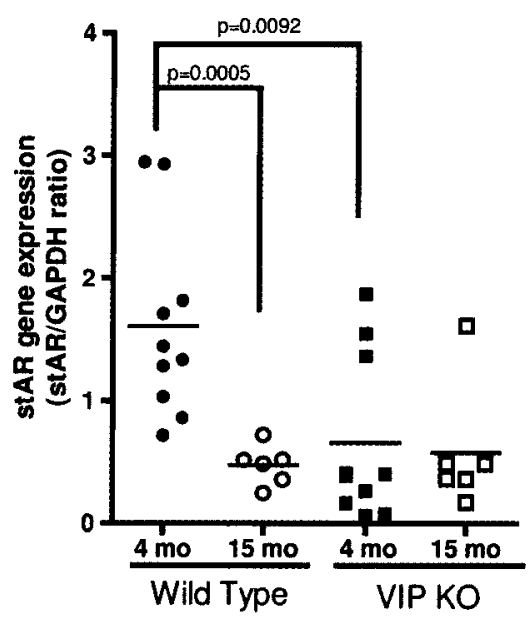

Q

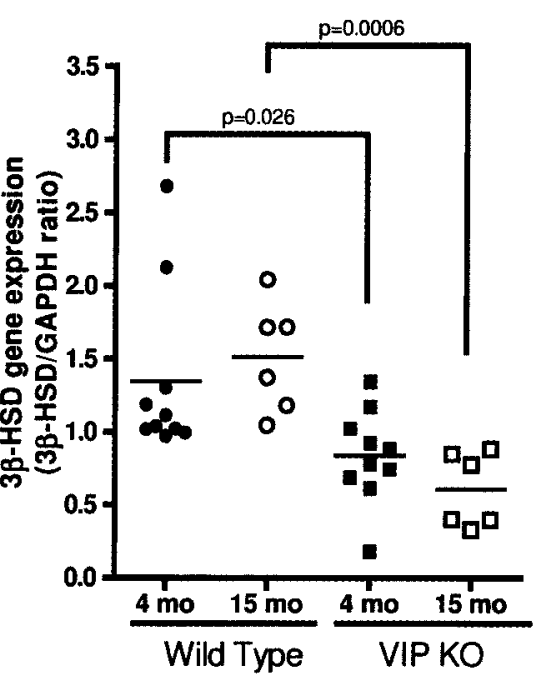

Figure 1: Comparaison des niveaux de testostérone et stéroïdogenèse pendant le vieillissement entre les souris sauvages et VIP-/-.

\section{(A) Niveaux de testostérone sériques chez les souris mâles sauvages et VIP-/-.}

Entre $4(n=8)$ et 15 mois ( $n=8)$, les niveaux de testostérone sériques diminuent de manière dramatique chez les animaux sauvages, alors que les niveaux restent bas et constants chez les souris VIP-/- ( $n=9$ pour les souris de 4 mois et $n=15$ pour les souris de 15 mois). Une étude statistique two-way ANOVA suivie d'un test ad hoc Bonferroni a montré que les différences entre les groupes génotypiques à 4 mois étaient significatives $(p<0.01)$.

\section{(B, C) Profile d'expression de StAR et 3B-HSD au cours du vieillissement.}

Les testicules des souris sauvages ( $n=5$ pour 4 mois et $n=3$ pour 15 mois) et VIP-/- ( $n=6$ pour 4 mois et $n=5$ pour 15 mois) ont été disséqués et préparés comme décrit dans la section Matériels et Méthodes. Les expériences de RT-PCR quantitatives ont révélé que les niveaux d'expression de StAR (B) sont significativement réduits chez les souris sauvages âgées de 15 mois, mais restent bas et constants chez les animaux VIP-/-. Des expériences similaires avec $3 B-H S D$ (C) n'ont révélé aucune différence de niveaux d'expression chez les souris sauvages, entre 4 et 15 mois. Chez les souris VIP-/-, comme pour les niveaux de StAR, les niveaux d'expression de 3ß-HSD sont bas et constants. La normalisation des données a été réalisée grâce à GAPDH. Les données présentées sur le graphique sont le résultat de deux expériences séparées. Une étude statistique two-way ANOVA suivie d'un test ad hoc Bonferroni a montré que les différences entre les groupes génotypiques à 4 mois étaient significatives $(p<0.01, p<0.01$ et $p<0.05)$ pour les niveaux de testostérone, StAR et $3 ß-H S D$ respectivement, alors que ces différences étaient significatives, à l'âge de 15 mois, seulement pour $3 ß-H S D(p<0.01)$. 


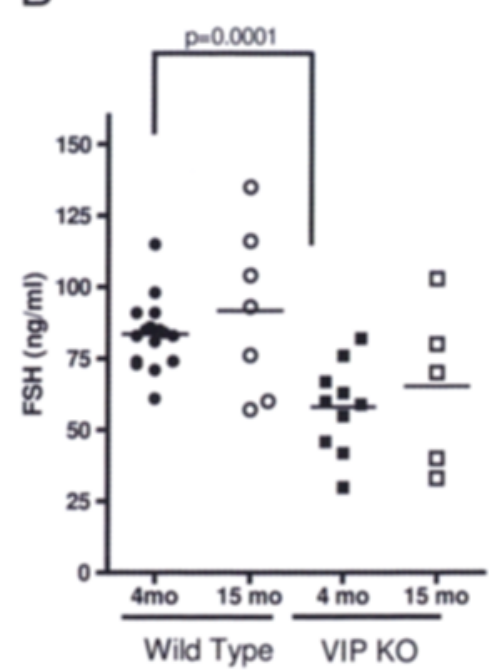

Figure 2 : Comparaison des niveaux de $L H(A)$ et FSH (B) sériques, au cours du vieillissement, chez les souris mâles sauvages et VIP-/.. Les niveaux de LHet FSH ont été mesurés comme décrit dans la section Matériels et Méthodes. Pour LH: $n=15$ souris sauvages et $n=9$ souris VIP-/-âgées de 4 mois, et $n=5$ souris sauvages et $n=5$ souris VIP/- âgées de 15 mois. Pour FSH : $n=16$ souris sauvages et $n=10$ souris VIP-/-âgées de 4 mois, et $n=7$ souris sauvages et $n=5$ souris VIP-/- âgées de 15 mois. Un test two-way ANOVA suivi d'un test ad hoc Bonferroni a montré que les différences entre les groupes génotypiques à 4 mois étaient significatives pour FSH $(p<0.01)$.
A

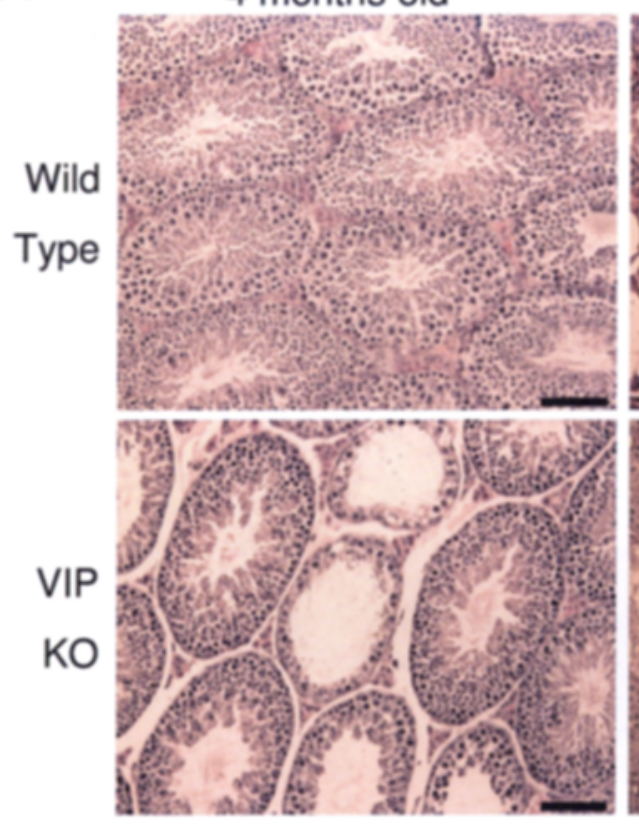

B

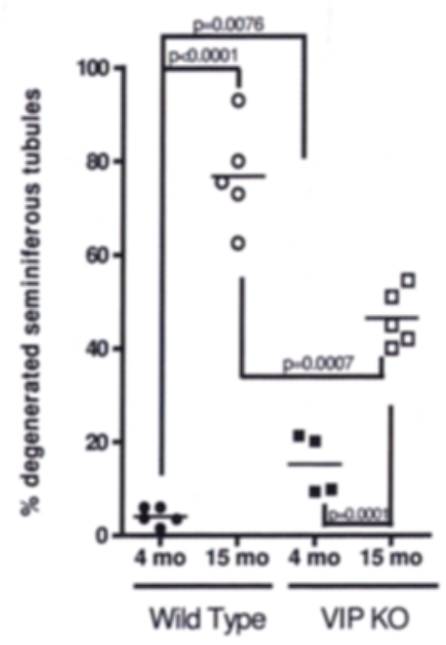

C

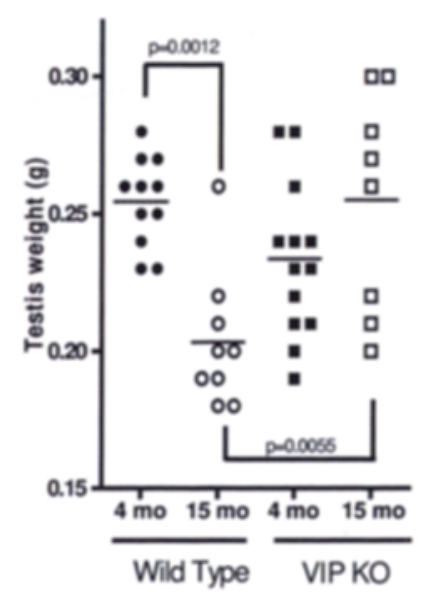

Figure 3 : Histopathologie testiculaire des souris sauvages et VIP-/- âgées de 4 et 15 mois.

(A) Les testicules des souris sauvages âgées de 4 mois ( $n=5)$ et 15 mois ( $n=5)$ et des souris VIP-/- âgées de 4 mois ( $n=4)$ et 15 mois $(n=8)$ ont été fixés dans $4 \%$ de paraformaldéhyde et préparés dans de la paraffine. Les sections $(6 \mu \mathrm{m})$ ont été réhydratées et colorées avec des solutions d'éosine et d'hématoxyline (barre de mesure, $100 \mu \mathrm{m}$ ). La double flèche montre la déplétion des cellules souvent observée au sein des tubules séminifères des souris sauvages âgées de 15 mois et la flèche noire rend compte de la présence de vacuolisations également retrouvées à une fréquence élevée au sein des tubules séminifères de ces animaux.

(B) A partir de sections testiculaires colorées à l'éosine et l'hématoxyline (n compris entre 4 et 8 pour chaque groupe), deux populations de tubules séminifères ont été discriminées : ceux "normaux" si l'architecture générale montrait une spermatogenèse normale, et ceux "dégénérés" s'ils montraient soit une déplétion d'une ou plusieurs couches de cellules germinales, ou des vacuolisations, ou bien les deux.

(C) Les testicules et épididymes des souris sauvages et VIP-/- âgés de 4 mois ( $n=11$ et $n=10$ respectivement) ont été disséqués et pesés, ainsi qu'à 15 mois ( $n=9$ et $n=7)$. Un test two-way ANOVA suivi d'un test ad hoc Bonferroni a montré que les différences entre les groupes génotypiques à 4 mois étaient significatives $(p<0.01)$ pour les tubules dégénérés. 
facteurs sont essentiels pour le maintien de la spermatogenèse $[12,15]$.

Il est donc possible que le phénotype que nous observons chez les souris mutantes VIP-/- soit la combinaison d'au moins deux évènements distincts : 1) une protection contre le vieillissement testiculaire résultant d'un niveau très bas de testostérone et par conséquent un niveau de formation de ROS réduit par rapport aux souris de type sauvage, et 2) une dégénérescence testiculaire parce que le testicule est soumis à des niveaux bas de testostérone et de FSH.

Le schéma du fonctionnement de l'axe hypothalamohypophysaire et son dérèglement chez les souris VIP -l- est rapporté à la Figure 4.
Parmi ses effets biologiques, le VIP est un facteur qui régule la croissance, la prolifération et la différenciation de nombreux types cellulaires [14, 7]. II est donc aussi plausible que le VIP puisse agir directement sur les cellules testiculaires et induire de tels effets dans ce tissu.

Le fait que la stéroïdogenèse testiculaire soit affectée chez les deux types de souris KO (VIP-/- et PACAP-1) laisse penser qu'il n'y a pas d'effet compensatoire entre ces neuropeptides. Cependant, le mécanisme est très probablement plus complexe et pourrait impliquer d'autres phénomènes. Afin de répondre à cette interrogation, nous avons récemment généré des souris doubles mutantes VIP/PACAP KO et la caractérisation de leur fertilité est en cours.

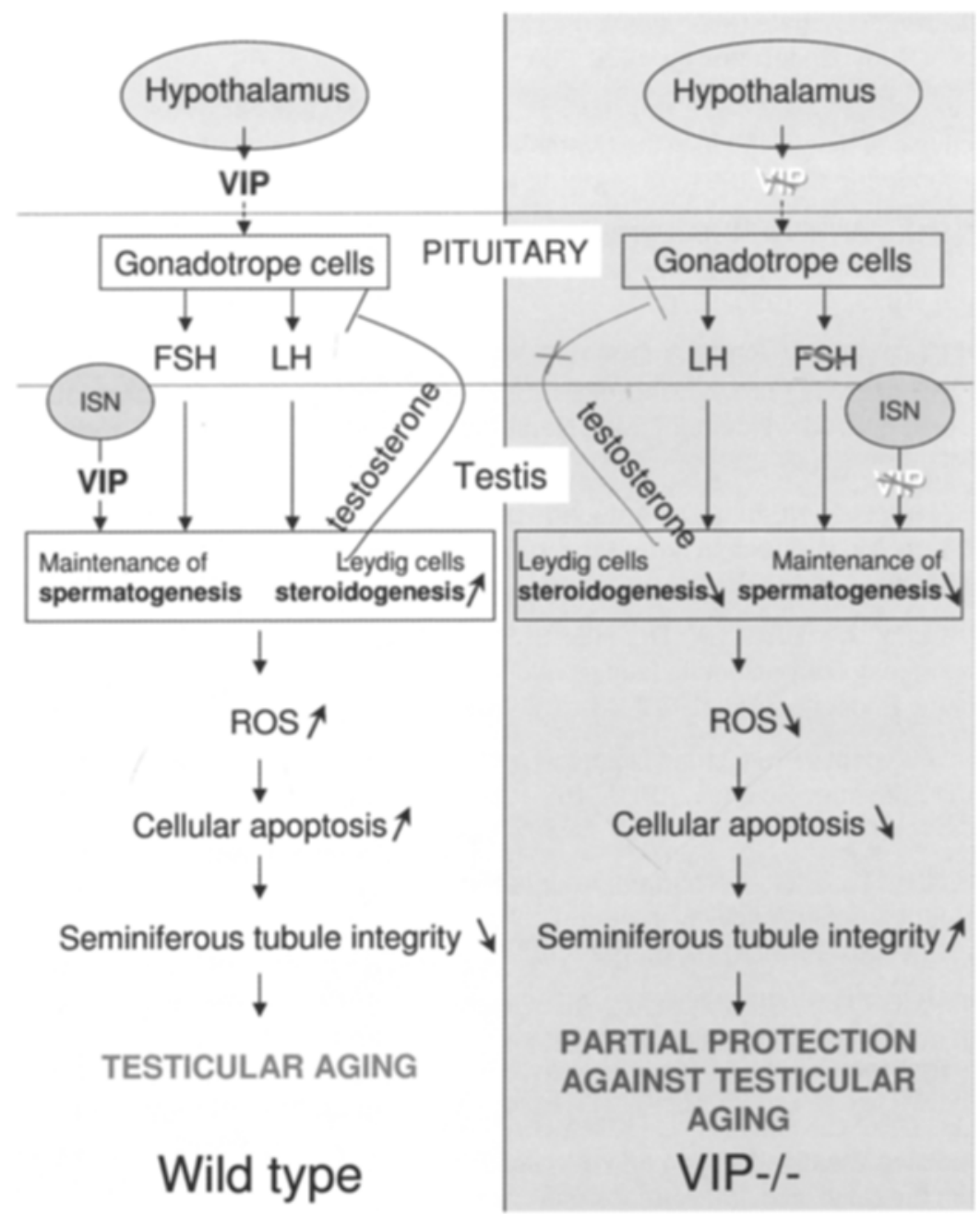

Figure 4 : Schéma bilan du fonctionnement de l'axe hypothalamo-hypophysaire et son dérèglement chez les souris VIP. 1-. Chez les animaux sauvages (panneau de gauche), la sécrétion de LH et FSH est stimulée par le VIP, au niveau pituitaire. Aussi, afin de médier ses actions locales, le VIP est sécrété au niveau des cellules de Leydig par des fibres VIPergiques spécifiques. De ce fait, la stéroïdogenèse des cellules de Leydig est stimulée. La genèse des radicaux libres, stimulée par le processus de stérö̈dogenèse lui-même, induit l'apoptose des cellules testiculaires, phénomène qui finalement mène au vieillissement testiculaire. Chez les animaux VIP-/- (panneau de droite), deux phénomènes ont lieu : 1) le niveau de production de radicaux libres (ROS) est réduit comparé aux souris sauvages car la stéroïdogenèse est diminuée, et 2) le niveau de maintien de la spermatogenèse est diminué à cause des bas niveaux de FSH et testostérone. En conséquence, la combinaison de ces deux évènements conduirait à une protection partielle contre le vieillissement testiculaire. 


\section{RÉFÉRENCES}

1. BRENNEMAN D.E., GLAZNER G., HILL J.M., HAUSER J., DAVIDSON A., GOZES I. : VIP neurotrophism in the central nervous system: multiple effectors and identification of a femtomolar-acting neuroprotective peptide. Ann. N.Y. Acad. Sci., 1998, 865 : 207-212.

2. EL-GEHANI F., TENA-SEMPERE M., HUHTANIEMI I. : Vasoactive intestinal peptide stimulates testosterone production by cultured fetal rat testicular cells. Mol. Cell. Endocrinol., 1998, $140: 175-178$.

3. KASSON B.G., LIM P., HSUEH A.J. : Vasoactive intestinal peptide stimulates androgen biosynthesis by cultured neonatal testicular cells. Mol. Cell. Endocrinol., 1986, $48: 21-29$.

4. KOVES K., GOTTSCHALL P.E., GORCS T., SCAMMELL J.G., ARIMURAA. : Presence of immunoreactive vasoactive intestinal polypeptide in anterior pituitary of normal male and long term estrogen-treated female rats : a light microscopic immunohistochemical study. Endocrinology, 1990, 126 : 17561763.

5. LAM K.S. : Vasoactive intestinal peptide in the hypothalamus and pituitary. Neuroendocrinology, 1991, 53 Suppl $1: 45-51$

6. LOREN I., EMSON P.C., FAHRENKRUG J. et al. : Distribution of vasoactive intestinal polypeptide in the rat and mouse brain. Neuroscience, 1979, 4 : 1953-1976.

7. MULLER J.M., LELIEVRE V., BECQ-GIRAUDON L., MEUNIER A.C. : VIP as a cell-growth and differentiation neuromodulator role in neurodevelopment. Mol. Neurobiol., $1995,10: 115-134$.

8. NAGY G., MULCHAHEY J.J., NEILL J.D. : Autocrine control of prolactin secretion by vasoactive intestinal peptide. Endocrinology, 1988, 122 : 364-366.

9. RESKO J.A., MALLEY A., BEGLEY D., HESS D.L. : Radioimmunoassay of testosterone during fetal development of the rhesus monkey. Endocrinology, 1973, 93 : 156-161.

10. SAID S.I., MUTT V. : Polypeptide with broad biological activity: isolation from small intestine. Science, 1970, $169: 1217-$ 1218.

11. SAID S.I., ROSENBERG R.N. : Vasoactive intestinal polypeptide : abundant immunoreactivity in neural cell lines and normal nervous tissue. Science, 1976, $192: 907-908$.

12. SIMONI M., WEINBAUER G.F., GROMOLL J., NIESCHLAG $E$. : Role of $\mathrm{FSH}$ in male gonadal function. Ann. Endocrinol. (Paris), 1999, 60 : 102-106.

13. SUH J.K., MUN K.H., CHO C.K., SHIN H.C., KIM Y.S., PARK T.C. : Effect of vasoactive intestinal peptide and acetylcholine on penile erection in the rat in vivo. Int. J. Impot. Res., 1995, $7: 111-118$

14. WASCHEK J.A. : Vasoactive intestinal peptide : an important trophic factor and developmental regulator? Dev. Neurosci., 1995, $17: 1-7$.

15. ZHANG F.P., PAKARAINEN T., POUTANEN M., TOPPARI J., HUHTANIEMI I. : The low gonadotropin-independent constitutive production of testicular testosterone is sufficient to maintain spermatogenesis. Proc. Natl. Acad. Sci. USA, 2003, $100: 13692-13697$.
Manuscrit reçu : janvier 2007 ; accepté janvier 2007.

Bourse SALF 2006.

\section{ABSTRACT}

Role of Vasoactive Intestinal Peptide (VIP) in
steroidogenesis and aging in mouse testis

Arnaud LACOMBE, Vincent LELIEVRE, Charles E. ROSELLI, Jean-Marc MULLER, James A. WASCHEK and Eric VILAIN

VIP (vasoactive intestinal peptide) neuropeptide has long been considered to be putative regulator of testicular functions. In vitro evidence suggests that VIP could play an important role in testosterone biosynthesis. However, the endogenous role of VIP on testicular functions remained to be demonstrated.

In C57BL/6 mice exhibiting complete disruption of the VIP gene, the authors observed that male fertility remained intact but serum testosterone levels were lower than those of WT littermates. At the age of 4 months, this phenotype was accompanied by reduced steroidogenesis due to inhibition of the expression of StAR (steroidogenic acute regulatory protein) and 3 BHSD (3ß-hydroxysteroid dehydrogenase) in the testis. In addition, serum levels of FSH (Follicle-stimulating hormone) but not LH (Luteinizing hormone) were reduced in young $\mathrm{KO}$ males. Testicular anatomy also revealed a subtle but significantly higher percentage of degenerated seminiferous tubules in 4month-old VIP-/- animals compared to WT.

In aging animals (15 months old), control males showed typical testicular aging including severe degeneration of seminiferous tubules, a dramatic decrease in serum testosterone levels and a reduction in StAR and 3B-HSD gene expression. In age-matched VIP-I- males, serum levels of testosterone and steroidogenic enzymes were still very low. Interestingly, in contrast with young mice, testicular degeneration at 15 months was significantly less severe marked in VIP-/- mice than in WT mice. Altogether, these results suggest that: 1) VIP is an important factor for regulating testosterone biosynthesis and $\mathrm{FSH}$ secretion and 2) VIP regulates testicular aging.

Key words : neuropeptide, testosterone biosynthesis, testicular aging, Luteinizing Hormone, Follicle-Stimulating Hormone 